\title{
Micropatterning of cells reveals chiral morphogenesis
}

\author{
Leo Q Wan 1,* Kacey Ronaldson², Mark Guirguis' and Gordana Vunjak-Novakovic 2,**
}

\begin{abstract}
Invariant left-right (LR) patterning or chirality is critical for embryonic development. The loss or reversal of $L R$ asymmetry is often associated with malformations and disease. Although several theories have been proposed, the exact mechanism of the initiation of the LR symmetry has not yet been fully elucidated. Recently, chirality has been detected within single cells as well as multicellular structures using several in vitro approaches. These studies demonstrated the universality of cell chirality, its dependence on cell phenotype, and the role of physical boundaries. In this review, we discuss the theories for developmental LR asymmetry, compare various in vitro cell chirality model systems, and highlight possible roles of cell chirality in stem cell differentiation. We emphasize that the in vitro cell chirality systems have great promise for helping unveil the nature of chiral morphogenesis in development.
\end{abstract}

\section{Left-right asymmetry in development and disease}

Organisms often adopt consistent left-right (LR) asymmetric positioning and morphology of internal organs, a phenomenon known as handedness or chirality. The chirality of biomolecules such as sugar and DNA has been recognized for a long time, but the origins of LR asymmetry in living organisms are not yet well understood. In general, the LR patterning is considered to follow four steps: (i) LR symmetry breaking by orienting the LR axis with respect to the anteroposterior and dorsoventral axes [1]; (ii) transferring initial chiral information into LR positions in a multicellular field; (iii) LR asymmetric expression of signaling molecules; and (iv) asymmetric morphogenesis of visceral organs induced

\footnotetext{
*Correspondence: wanq@rpi.edu; gv2131@columbia.edu

'Department of Biomedical Engineering, Rensselaer Polytechnic Institute, Troy, NY 12180, USA

²Department of Biomedical Engineering, Columbia University, New York, NY 10027, USA
}

by these molecules [2-4]. Proper LR patterning requires reliable breaking of LR symmetry at early developmental stages as well as the transmission and amplification of LR signals at later stages. Defects in any of the four steps may lead to severe outcomes in laterality. For instance, during the asymmetric gene expression, the midline structure functions as a barrier and keeps left-sided signals from affecting the right side, and vice versa [5]. Midline defects result in disturbances of normal laterality.

Abnormality in LR asymmetry is also closely associated with disease. First, abnormality in LR signaling often leads to malformations, including situs ambiguus (one or more organs in the mirrored position) and situs inversus (all internal organs in mirrored positions). These defects may result from heritable genetic diseases such as Kartagener syndrome, or prenatal exposure to teratogens [6]. Maternal diseases such as diabetes can also trigger laterality defects $[7,8]$.

Second, many diseases are associated with LR asymmetry $[9,10]$. The incidence of cancer significantly changes with laterality, with stronger occurrence of lung, testis, and ovarian cancer on the right side, and breast cancer and melanoma on the left side [9]. The upper limb malformations associated with Holt-Oram syndrome are also more common on the left side [11]. Detailed analyses showed that the position-dependent incidence of disease may not be necessarily associated with asymmetric organ mass or personal handedness and therefore remains unexplained [12].

Third, there is a strong correlation between breast cancer and abnormal cerebral asymmetry [13]. Altogether, these findings suggest that disease and abnormal LR asymmetry are closely related and may share common developmental origins, whether environmental or genetic [12-15]. Therefore, it is of great scientific interest and clinical significance to investigate the LR asymmetry in development and disease.

We focus on recent in vitro studies of LR asymmetry, within the overall context of LR patterning in development. Starting from the unsolved problems in two current models of LR symmetry breaking, we discuss in vitro studies of cell chirality, and their possible applications in vivo. 


\section{LR symmetry breaking in development}

The initiation of LR asymmetry in development, also known as LR symmetry breaking, is one of the most intriguing problems in developmental biology. While it is widely believed that LR patterning is ultimately derived from the chirality of biomolecules, the exact mechanism, timing, and location of symmetry breaking are still being debated. The central question is how the biophysical molecular chirality is converted into LR asymmetric gene expression during development. Two important models the primary cilium model and the voltage gradient model - can each provide an explanation, but they differ about how and when the molecular chirality gives rise to positional information.

In the primary cilium model (Figure 1a), the symmetry breaking is considered to occur at the ventral node at the late neural-fold stage of mouse development. The node cells are well organized and have beating primary cilia on their membranes, which drive a leftward flow, leading to the LR asymmetry of gene expression of Nodal and other proteins [16-18]. The LR asymmetry breaking via primary cilia has three essential characteristics: (i) a consistent posterior tilt of cilia; (ii) synchronized beating; and (iii) unidirectional cilia rotation (that is, in the counterclockwise direction when viewed from the ventral side). The posterior orientation of cilia and concerted beating are believed to relate to the interaction between node cells, possibly through planar cell polarity [19-21]. However, the mechanism by which cilia beat in a unidirectional fashion remains unclear. In addition, evidence suggests that the primary cilia model may not play equally important roles in all cases [22-24].

In snail, fly, chick and pig, cilia are not present during LR development. In addition, several mutants have abnormal or no ciliary motion, but their LR asymmetric body plan is found to be normal [24-26]. These suggest that ciliary motion is not necessarily required for LR patterning, and that other mechanisms might be responsible for LR symmetry breaking.

A second mechanism involves an electrical voltage gradient (Figure 1b) that transports small LR determinants such as serotonin to one side of the body, where they initiate asymmetric gene expression of Nodal and other proteins. The voltage gradient, determined by asymmetric localization of ion channels, was found as early as the two-cell or four-cell cleavage stages in the Xenopus and chick embryos $[27,28]$. In addition, cell asymmetric migration at the Hensen's node of chick embryos was found to be a downstream event for transient depolarization of membrane potential on the left side mediated by $\mathrm{H}^{+} / \mathrm{K}^{+}$ATPase activity.

It is believed that the ion channel mRNAs and proteins are directionally transported by the involvement of chiral 'F' molecules so that ion transporters are mostly localized

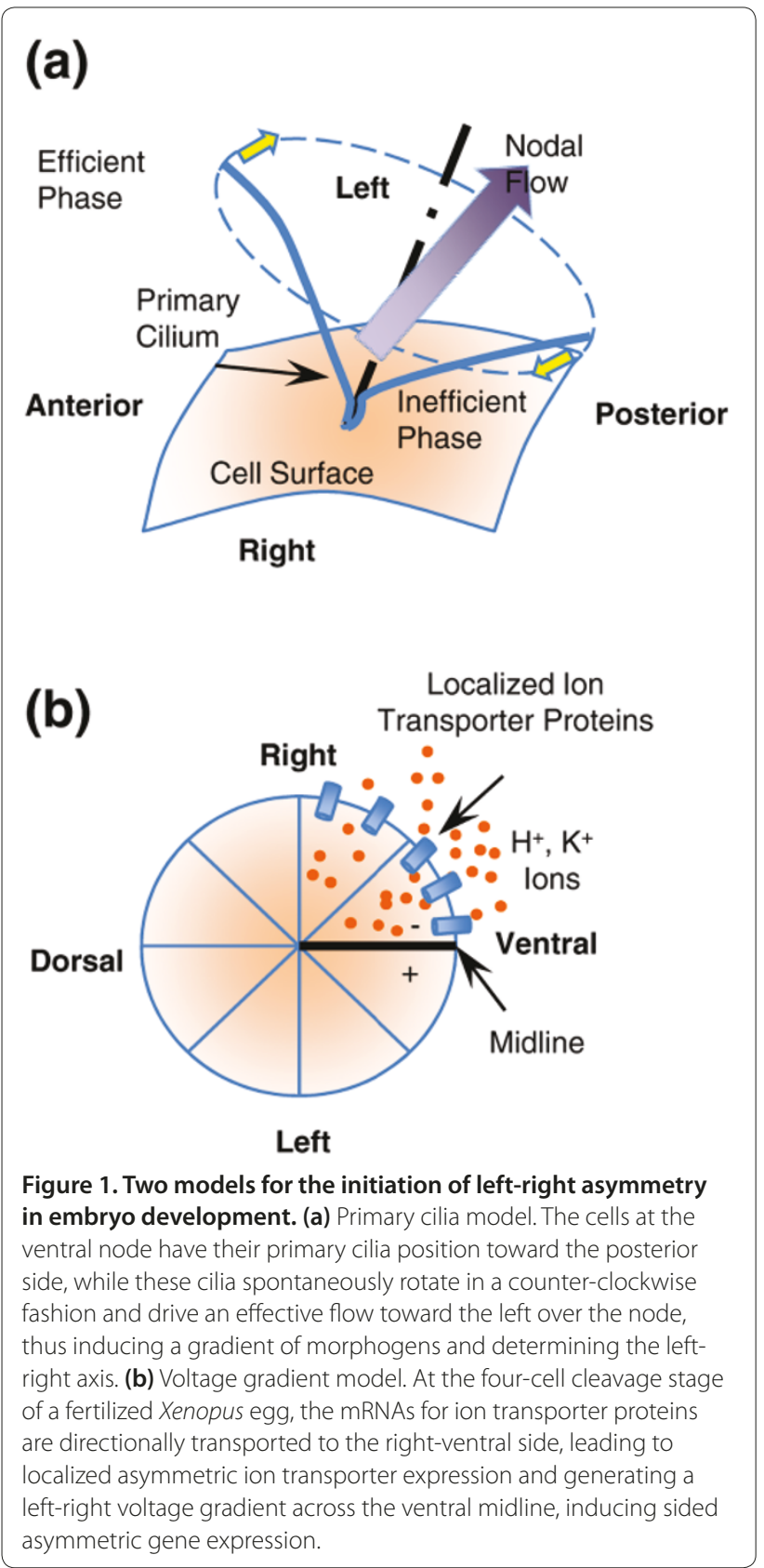

at the right-ventral side. The concept of ' $\mathrm{F}$ ' molecules was first presented by Wolpert and colleagues [1], and these molecules have three mutually perpendicular axes that follow the anteroposterior axis, the dorsoventral axis, and the LR axis. The nature of the ' $F$ ' molecule is largely unknown. A cytoskeletal origin of asymmetry was recently demonstrated by finding the pre-existence of chirality of actin cytoskeleton wrapped around the cortex of Xenopus eggs [29]. In addition, the actin/tubulin network was conjectured to fulfill the function of ' $F$ ' molecules, with actin aligned at the bottom and microtubules on the top along the LR direction, with the directional transport 
along microtubules defining the LR axis [4]. However, this cytoskeleton network model remains to be characterized and validated for the orientation of the LR axis with respect to the other two axes of the early embryo.

In general, the breaking of LR symmetry has been associated with the function of chiral cellular structures, such as the ventral node in mice. Importantly, chiral structures must be organized in a specific fashion so that their collective behavior allows generation of significant biophysical signals that can be translated into local asymmetric gene expression and subsequently amplified into the difference between the LR sides. Two questions are of fundamental interest in this regard: whether chirality is a fundamental property of the cell, and how the chirality of single cells is translated into multicellular chiral morphogenesis. It will be greatly helpful if these questions can be addressed in in vitro systems, where asymmetric biophysical and biochemical cues can be excluded.

\section{In vitro cell chirality}

The chirality has been observed in several cellular systems [30-32]. For instance, bacterial colonies can develop chiral morphology with branches twisted in the same handedness [30]. This behavior is believed to be derived from the chirality of the flagella that propel the cell body and induce a defined handedness of rotation. The neurite outgrowth of hippocampal explants has a rightward bias, and turns clockwise on two-dimensional substrates [31,32]. Such turning has been shown to be driven by the autonomous right-screw rotation of growth cone filopodia, possibly through the interactions between myosins ( $\mathrm{Va}$ and $\mathrm{Vb}$ ) and filamentous actins.

It was not until recently that intrinsic cell chirality has been studied in a well-controlled and highly repeatable fashion and detected in almost all cell types [33-35]. We will review recent research progress on cell chirality at the levels of single cells as well as multiple cells. In these in vitro systems, no node, cilia or fluid flow is required to establish consistent asymmetry, indicating that chirality is a fundamental intrinsic cellular property.

Chirality at the single cell level was clearly demonstrated by $\mathrm{Xu}$ and colleagues [33] through studying the migration of sparsely seeded neutrophil-like differentiated HL-60 (dHL-60) cells (Figure 2). It was found that upon the induction of polarization by a uniform chemoattractant (f-Met-Leu-Phe (fMLP)), the cells primarily migrated in the direction defined by the axis from the middle of the nucleus to the centrosome, but had a tendency to move toward the left side. The authors proposed that the chiral structure of the centrosome could be responsible for the observed single cell chirality, and that the centrosome could polarize the cell and subsequently determine its directional response to

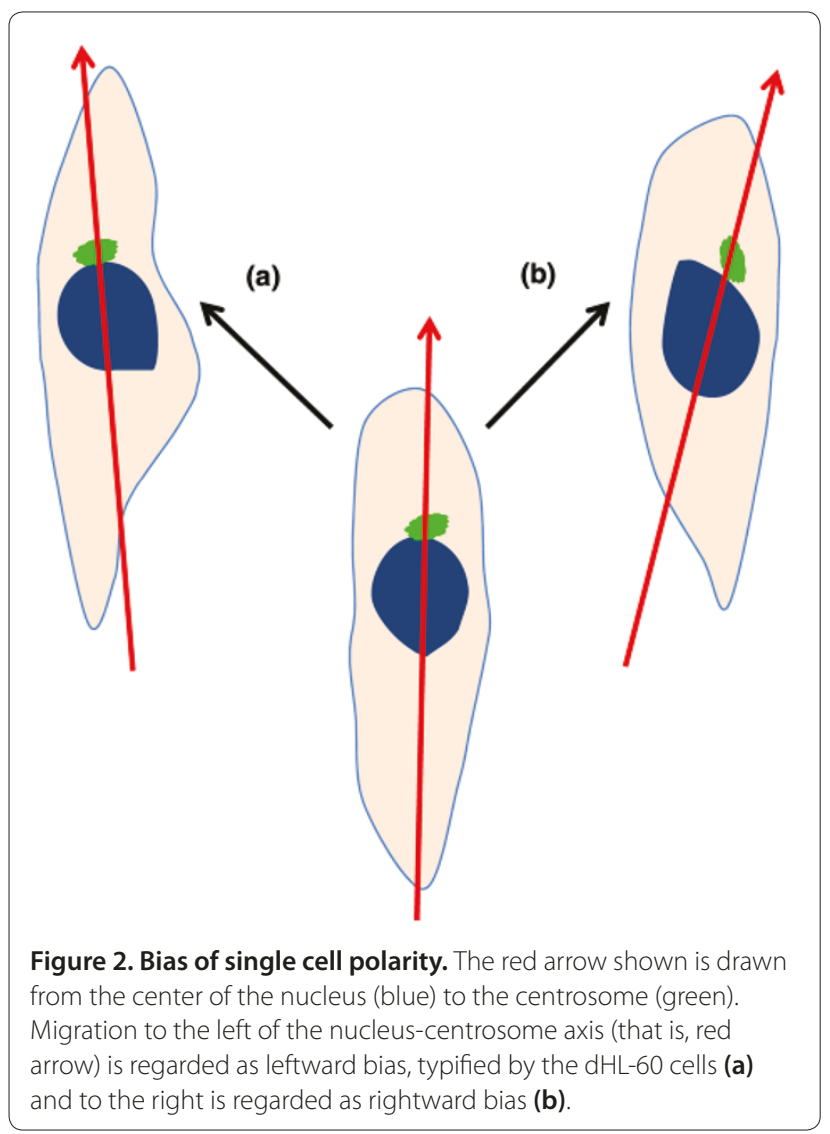

chemical stimuli. The cell chirality could be observed by inducing neutrophils to polarize without creating spatial cues and by studying single cell polarization in conditions free of the effects of neighboring cells. The authors defined cell chirality as either the leftward or rightward bias, with respect to two predefined cellular axes: one axis defined by the cells' attachment to the substrate and the other axis defined by the centrosomes' relation to the nuclei. A leftward bias was observed for dHL-60 cells and found to be microtubule dependent. Inspired from studies in neurons and astrocytes, the Cdc42/Par6 polarity pathway was investigated for specific mechanisms of establishing cell chirality. Upstream disruption of the pathway (that is, interfering Par6, Cdc42, or phosphatidylinositol $(3,4,5)$-trisphosphate) prevented the establishment of polarity altogether, while downstream disruptions (that is, interfering protein kinase $\mathrm{C}-\zeta$ or dynein) were only found to randomize the chirality. Surprisingly, constitutive activation of glycogen synthase kinase-3 $\beta$ (GSK3 $\beta$ ), located in the middle of the proposed Cdc42/Par6 polarity pathway, was able to reverse the bias of fMLPinduced polarity rather than just randomize it. Recently, this system was used to demonstrate the non-ciliary role of microtubule proteins in LR patterning across biological kingdoms [36]. Mutation of tubulin was shown to alter 
LR patterning in plants, nematodes and frogs, as well as human cells in culture.

The establishment of chirality at a multicellular level has also been related to the intrinsic cell chirality. We previously investigated the establishment of the LR axis by characterizing multicellular alignment and migration in response to micropatterned appositional boundaries (Figure 3) [34,37]. By using micropatterning techniques, a cell monolayer was formed within geometries, which imposed boundary conditions on cells and thus forced them to make the LR decision. A prerequisite to forming this chirality was the geometric imposition of two opposing boundaries within a distance up to several hundred micrometers, such as those found in geometries composed of linear strips and rings but not in square or circle geometries. Primary use of ring geometry allowed for the use of an image-based algorithm, based on the overall alignment of individual cells with respect to the circumferential direction, to determine the multicellular chirality as clockwise $(\mathrm{CW})$ or counter-clockwise $(\mathrm{CCW})$.

This LR decision was apparent at a multicellular level, and specific to cell phenotype. Most investigated cells (for example, endothelial cells, osteoblasts, adult mesenchymal stem cells, and fibroblasts) displayed a CW bias, except for two myoblast cell lines, derived from mouse and human skeletal muscles, which were found to have a CCW bias. These results suggest that cell chirality might alter during the differentiation of stem cell function. Therefore, it will be necessary to investigate the chirality of the cells relevant to LR asymmetry development, and examine effects of important biochemical and biophysical LR signals.

Investigations into the mechanism behind multicellular chirality revealed the role of actin in the establishment of $\mathrm{CCW}$ cell lines, as revealed by the reversal of $\mathrm{CCW}$ chirality to $\mathrm{CW}$ chirality upon the introduction of actininterfering drugs. Surprisingly, it was found that microtubule-destabilizing drugs had no effect on the establishment of CCW or CW chirality. Thus, while the establishment of a directional bias is still undetermined, there is a clear role for functional actin in establishing the CCW alignment. In addition, the cells were found to have a consistent polarization, with their centrosome (rather than the nuclei) positioned closely to geometrical boundaries [38], and a directional cell migration along the boundary. Using the same definition of three axes of $\mathrm{Xu}$ and colleagues [33], the CCW alignment on rings can be considered as a cellular 'leftward' bias, and CW as a 'rightward' bias.

Recently, Chen and colleagues [35] also were able to observe multicellular chirality at a larger scale (that is, several centimeters) on two-dimensional surfaces through the use of micro-fabrication techniques (Figure 4). They found that culturing vascular mesenchymal cells on

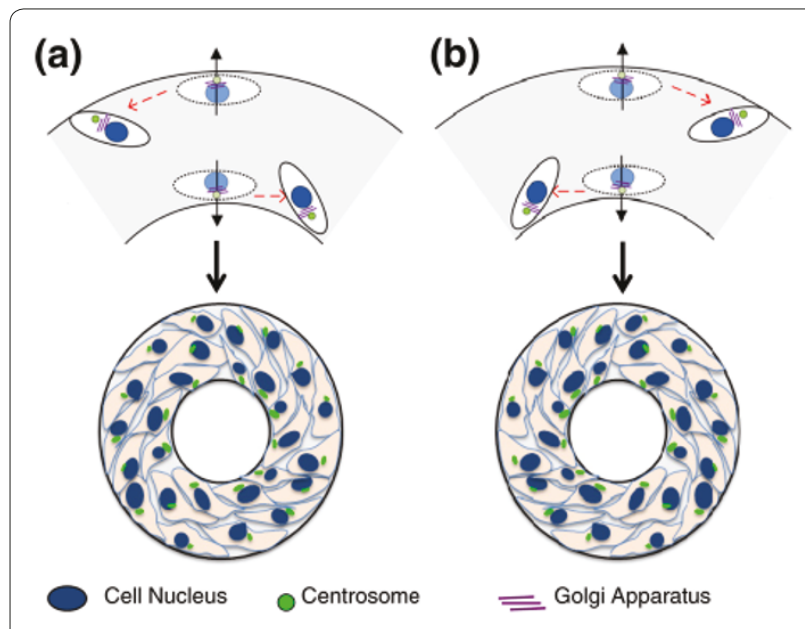

Figure 3. Left-right asymmetry on micropatterned surfaces. The cells are polarized at the boundary by positioning their centrosomes (green) and Golgi apparatuses (purple) closer to each boundary than nuclei (blue), while forming chiral alignment. (a) Polarity and chirality of muscle cells on micropatterned surfaces. The leftward bias of muscle cells on appositional boundaries creates the observed counter-clockwise (CCW) cell alignment. (b) Schematic of polarity and chirality of endothelial cells on micropatterned surfaces. The rightward bias of cell migration creates the observed clockwise (CW) alignment.

substrates containing alternating cell-adhesive coated fibronectin lanes and non-adhesive coated polyethylene glycol lanes resulted in a highly organized chiral pattern. The cells initially attached to fibronectin-coated lanes with a similar chiral alignment found by Wan and colleagues [34], and subsequently expanded to the entire surface with polyethylene glycol degradation, forming a rightward bias of an approximately $20^{\circ}$ angle between grooves and aligned cells. The accumulation of stress fibers upon encountering substrate interfaces was speculated to induce mechanical cues that lead to the formation of chiral patterns aligned diagonally to these interfaces. This hypothesis was confirmed in inhibition studies with the nonmuscle myosin-II inhibitor blebbistatin, and the Rho-associated kinase (ROCK) inhibitor Y27632.

A reaction-diffusion mathematical model was developed to describe possible effects of two morphogens: bone morphogenesis protein (BMP)-2, a slowly diffusing activator, and matrix $\gamma$-carboxyglutamic acid protein (MGP), a rapidly diffusing inhibitor of BMP [39]. Within a two-dimensional domain, they created a spatiotemporal gradient of chemicals. The cells were assumed to respond to the gradient of morphogens with a consistent directional bias that was responsible for the chirality of pattern formation. However, it needs to be experimentally validated whether and by which mechanisms these two morphogens can induce a reliable bias relative to the chemical gradient axes. 


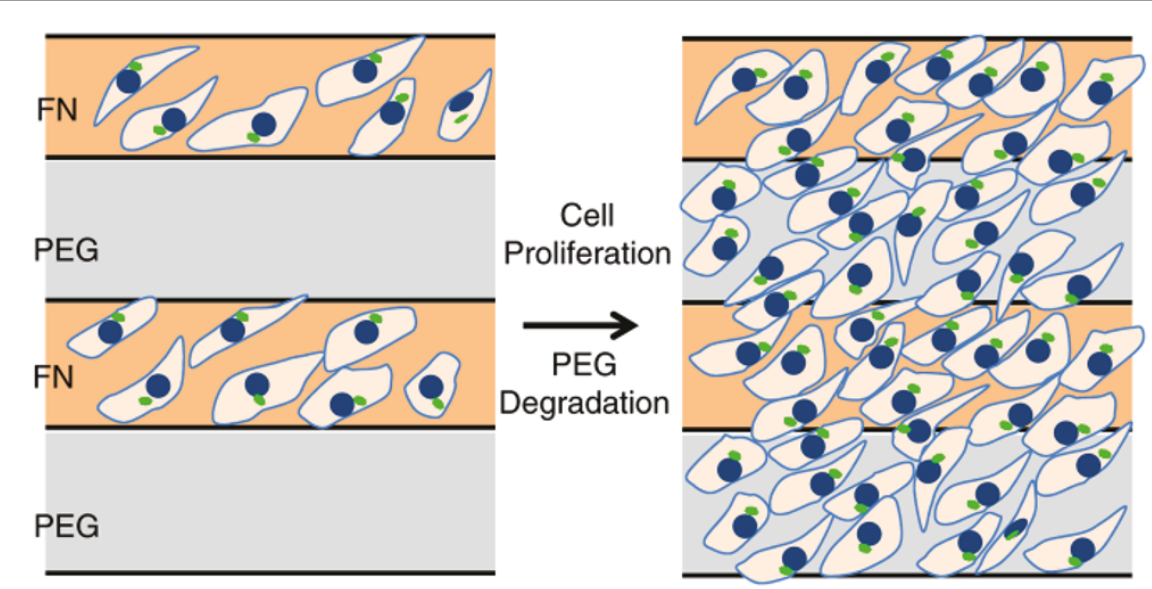

Figure 4. Propagation of cell chirality with loss of geometric control. Cells preferentially attach to the fibronectin (FN) domain immediately after plating onto alternating fibronectin/polyethylene glycol (PEG) lanes, and they form a biased alignment within the FN domain. With PEG degradation and cell proliferation, the cells migrate out of the FN domain but maintain chirality over the entire surface.

In summary, all the in vitro cell chirality systems rely on definite cell polarization either transiently determined upon the exposure to chemoattractants, or induced by physical boundaries, and the chirality is detected as a result of biased cell movement in a direction perpendicular to cell polarization. The single cell system [33] presents the first direct demonstration of the existence of chirality of single cells, but it requires cell centrosome and nucleus live imaging and is currently limited to only one cell type, dHL-60. The micropatterning system by Wan and colleagues [34], on the contrary, demonstrated, with a variety of cells in a highly reliable fashion, that multicellular chiral structures can emerge from a homogeneous cell population within well-controlled microscale boundaries. Chen and colleagues [35] further showed that such an initial cell chiral alignment could be propagated into a larger scale if cells are allowed to migrate out of the boundaries. Altogether, these studies suggest that most, if not all, cells are chiral in nature, and single cell chirality can manifest into multicellular chiral morphogenesis with well-controlled boundaries, even at a large scale.

\section{Possible roles of cell chirality in LR asymmetry}

All these in vitro cell chirality systems suggest that chiral morphogenesis can be generated without specific embryonic structures such as ventral node or even beating cilia. Most, if not all, cells are intrinsically chiral, just like what was found with Xenopus eggs [29]. Therefore, these in vitro studies provide further support for cytoplasmic models, such as directional transport in fertilized eggs in the voltage gradient model. Furthermore, in vitro studies demonstrate that, under certain conditions, cell chirality will appear as biases in cellular function: migration and alignment. These new findings may provide alternative explanations for LR embryonic development.

We believe that advances in the understanding of cell chirality in in vitro systems could potentially help unveil the mechanism of in vivo LR asymmetry development $[37,40]$. First, the intrinsic cell chirality may utilize the same cell machinery that is responsible for LR symmetry breaking. The current models for early LR development (that is, nodal flow and voltage gradient) still have essential unanswered questions, which could be addressed by studying cell machinery involved in intrinsic cell chirality. Second, it is possible that the intrinsic cell chirality may contribute to other LR asymmetry events such as heart tube and gut looping, and brain asymmetry [22]. Third, boundaries might direct the establishment of chiral structures. Interestingly, biased cell migration has been found around the Hensen's node (equivalent to the ventral node in mice) in chicken development [41].

Interestingly, drugs regulating cell chirality resemble those affecting LR asymmetry. Actin function was critical for cell chirality on patterned surfaces. Many asymmetric breaking events are related to actin function, such as asymmetric cell division, chiral alignment of embryonic cells at early stages of development, and in vitro actin shear structure induced in the Xenopus egg. In the snail Lymnaea stagnalis, the interference of actin dynamics, but not microtubules, alters the chirality of early dextral embryos [42]. In the hindgut looping of Drosophila, the mutation of myosin ID, an actin motor, was found to reverse chirality of hindgut looping [43-45]. In Caenorhabditis elegans, the LR asymmetry was found to associate with actin and cortical forces and be regulated by noncanonical signaling pathways [46]. 
The recent progress in the in vitro cell chirality may greatly accelerate research in LR asymmetry during development. An in vitro cell chirality system allows for quick assessment of effects and mechanisms of relevant drugs and growth factors in a well-controlled manner, by excluding possible confounding factors.

However, it is important to point out that it is still not clear when and how cell chirality is exactly utilized during the development. In particular, the cells on a twodimensional substrate may behave quite differently from those in embryonic development. Cellular microenvironments, including soluble factors and extracellular matrix, need to be better mimicked in in vitro systems [47-49] before they can be utilized in LR asymmetry research in embryonic development.

\section{Conclusion}

In vitro cell chirality may create a new paradigm to study the role of intracellular and intercellular machinery in LR asymmetry breaking in development. Whether and to what extent the intrinsic cell chirality can be used to explain the LR asymmetry events are intriguing questions to be addressed in future work.

This article is part of a thematic series on Physical influences on stem cells edited by Gordana Vunjak-Novakovic. Other articles in the series can be found online at http://stemcellres.com/series/physical

\section{Abbreviations}

CW, clockwise; CCW, counter-clockwise; dHL-60, differentiated HL-60; fMLP, f-Met-Leu-Phe; LR, left-right.

\section{Competing interests}

The authors declare that they have no competing interests; however, Columbia University is applying for a patent related to a technology for measuring cell chirality with micro-patterns.

\section{Acknowledgments}

We gratefully acknowledge NIH support of our work (grant EB002520).

Published: 3 April 2013

\section{References}

1. Brown NA, Wolpert $L$ : The development of handedness in left/right asymmetry. Development 1990, 109:1-9.

2. Levin M: Is the early left-right axis like a plant, a kidney, or a neuron? The integration of physiological signals in embryonic asymmetry. Birth Defects Res C Embryo Today 2006, 78:191-223.

3. Hamada H, Meno C, Watanabe D, Saijoh Y: Establishment of vertebrate left-right asymmetry. Nat Rev Genet 2002, 3:103-113.

4. Levin M, Mercola M: The compulsion of chirality: toward an understanding of left-right asymmetry. Genes Dev 1998, 12:763-769.

5. Tabin CJ: The key to left-right asymmetry. Cell 2006, 127:27-32.

6. Aylsworth AS: Clinical aspects of defects in the determination of laterality. Am J Med Genet 2001, 101:345-355.

7. Slavotinek A, Hellen E, Gould S, Coghill SB, Huson SM, Hurst JA: Three infants of diabetic mothers with malformations of left-right asymmetry - further evidence for the aetiological role of diabetes in this malformation spectrum. Clin Dysmorphol 1996, 5:241-247.

8. Ewart-Toland A, Yankowitz J, Winder A, Imagire R, Cox VA, Aylsworth AS, Golabi M: Oculoauriculovertebral abnormalities in children of diabetic mothers. Am J Med Genet 2000, 90:303-309.
9. Roychoudhuri R, Putcha V, Moller H: Cancer and laterality: a study of the five major paired organs (UK). Cancer Causes Control 2006, 17:655-662.

10. Paulozzi LJ, Lary JM: Laterality patterns in infants with external birth defects. Teratology 1999, 60:265-271.

11. Smith AT, Sack GH Jr, Taylor GJ: Holt-Oram syndrome. J Pediatr 1979, 95:538-543.

12. Wilting J, Hagedorn M: Left-right asymmetry in embryonic development and breast cancer: common molecular determinants? Curr Med Chem 2011, 18:5519-5527.

13. Sandson TA, Wen PY, LeMay M: Reversed cerebral asymmetry in women with breast cancer. Lancet 1992, 339:523-524.

14. Klar AJ: A hypothesis: breast cancer predisposition and brain hemispheric laterality specification likely share a common genetic cause. Breast Dis 2011, 33:49-52.

15. Klar AJ: Support for the selective chromatid segregation hypothesis advanced for the mechanism of left-right body axis development in mice. Breast Dis 2008, 29:47-56.

16. Hirokawa N, Tanaka Y, Okada Y, Takeda S: Nodal flow and the generation of left-right asymmetry. Cell 2006, 125:33-45.

17. Okada Y, Takeda S, Tanaka Y, Belmonte JC, Hirokawa N: Mechanism of nodal flow: a conserved symmetry breaking event in left-right axis determination. Cell 2005, 121:633-644

18. Nonaka S, Tanaka Y, Okada Y, Takeda S, Harada A, Kanai Y, Kido M, Hirokawa N: Randomization of left-right asymmetry due to loss of nodal cilia generating leftward flow of extraembryonic fluid in mice lacking KIF3B motor protein. Cell 1998, 95:829-837.

19. Antic D, Stubbs JL, Suyama K, Kintner C, Scott MP, Axelrod JD: Planar cell polarity enables posterior localization of nodal cilia and left-right axis determination during mouse and Xenopus embryogenesis. PLoS One 2010, 5:e8999.

20. Hashimoto M, Shinohara K, Wang J, Ikeuchi S, Yoshiba S, Meno C, Nonaka S, Takada S, Hatta K, Wynshaw-Boris A, Hamada H: Planar polarization of node cells determines the rotational axis of node cilia. Nat Cell Bio/ 2010, 12:170-176.

21. Song H, Hu J, Chen W, Elliott G, Andre P, Gao B, Yang Y: Planar cell polarity breaks bilateral symmetry by controlling ciliary positioning. Nature 2010, 466:378-382.

22. Speder P, Petzoldt A, Suzanne M, Noselli S: Strategies to establish left/right asymmetry in vertebrates and invertebrates. Curr Opin Genet Dev 2007 17:351-358.

23. Aw S, Levin M: Is left-right asymmetry a form of planar cell polarity? Development 2009, 136:355-366.

24. Vandenberg LN, Levin M: Far from solved: a perspective on what we know about early mechanisms of left-right asymmetry. Dev Dyn 2010, 239:3131-3146.

25. Serluca FC, Xu B, Okabe N, Baker K, Lin SY, Sullivan-Brown J, Konieczkowski DJ, Jaffe KM, Bradner JM, Fishman MC, Burdine RD: Mutations in zebrafish leucine-rich repeat-containing six-like affect cilia motility and result in pronephric cysts, but have variable effects on left-right patterning. Development 2009, 136:1621-1631.

26. Kishimoto N, Cao Y, Park A, Sun Z: Cystic kidney gene seahorse regulates cilia-mediated processes and Wnt pathways. Dev Cell 2008, 14:954-961.

27. Levin M, Thorlin T, Robinson KR, Nogi T, Mercola M: Asymmetries in $\mathrm{H}+$ / $\mathrm{K}+$-ATPase and cell membrane potentials comprise a very early step in left-right patterning. Cell 2002, 111:77-89.

28. Aw S, Adams DS, Qiu D, Levin M: H,K-ATPase protein localization and Kir4.1 function reveal concordance of three axes during early determination of left-right asymmetry. Mech Dev 2008, 125:353-372.

29. Danilchik MV, Brown EE, Riegert K: Intrinsic chiral properties of the Xenopus egg cortex: an early indicator of left-right asymmetry? Development 2006, 133:4517-4526.

30. Ben-Jacob E, Cohen II, Shochet O, Tenenbaum A, Czirok A, VicsekT: Cooperative formation of chiral patterns during growth of bacterial colonies. Phys Rev Lett 1995, 75:2899-2902.

31. Heacock AM, Agranoff BW: Clockwise growth of neurites from retinal explants. Science 1977, 198:64-66.

32. Tamada A, Kawase S, Murakami F, Kamiguchi H: Autonomous right-screw rotation of growth cone filopodia drives neurite turning. J Cell Bio/ 2010, 188:429-441.

33. Xu J, Van Keymeulen A, Wakida NM, Carlton P, Berns MW, Bourne HR: Polarity reveals intrinsic cell chirality. Proc Natl Acad Sci U S A 2007, 104:9296-9300. 
34. Wan LQ, Ronaldson K, Park M, Taylor G, Zhang Y, Gimble JM, Vunjak-Novakovic G: From the Cover: Micropatterned mammalian cells exhibit phenotypespecific left-right asymmetry. Proc Natl Acad Sci U S A 2011, 108:12295-12300

35. Chen TH, Hsu JJ, Zhao X, Guo C, Wong M, Huang Y, Li Z, Garfinkel A, Ho CM, Tintut $Y$, Demer LL: Left-right symmetry breaking in tissue morphogenesis via cytoskeletal mechanics. Circ Res 2012, 110:551-559.

36. Lobikin M, Wang G, Xu J, Hsieh YW, Chuang CF, Lemire JM, Levin M: Early, nonciliary role for microtubule proteins in left-right patterning is conserved across kingdoms. Proc Natl Acad Sci U S A 2012, 109:12586-12591.

37. Wan LQ, Vunjak-Novakovic G: Micropatterning chiral morphogenesis. Commun Integr Biol 2011, 4:745-748.

38. Desai RA, Gao L, Raghavan S, Liu WF, Chen CS: Cell polarity triggered by cell-cell adhesion via E-cadherin. J Cell Sci 2009, 122:905-911.

39. Garfinkel A, Tintut Y, Petrasek D, Bostrom K, Demer LL: Pattern formation by vascular mesenchymal cells. Proc Natl Acad Sci U S A 2004, 101:9247-9250

40. McSheene JC, Burdine RD: Examining the establishment of cellular axes using intrinsic chirality. Proc Natl Acad Sci U S A 201 1, 108:12191-12192.

41. Gros J, Feistel K, Viebahn C, Blum M, Tabin CJ: Cell movements at Hensen's node establish left/right asymmetric gene expression in the chick. Science 2009, 324:941-944

42. Shibazaki Y, Shimizu M, Kuroda R: Body handedness is directed by genetically determined cytoskeletal dynamics in the early embryo. Curr Biol 2004, 14:1462-1467.
43. Spéder P, Adám G, Noselli S: Type ID unconventional myosin controls leftright asymmetry in Drosophila. Nature 2006, 440:803-807.

44. Hozumi S, Maeda R, Taniguchi K, Kanai M, Shirakabe S, Sasamura T, Spéder P, Noselli S, Aigaki T, Murakami R, Matsuno K: An unconventional myosin in Drosophila reverses the default handedness in visceral organs. Nature 2006, 440:798-802.

45. Taniguchi K, Maeda R, Ando T, Okumura T, Nakazawa N, Hatori R, Nakamura M, Hozumi S, Fujiwara H, Matsuno K: Chirality in planar cell shape contributes to left-right asymmetric epithelial morphogenesis. Science 2011, 333:339-341.

46. Pohl C, Bao Z: Chiral forces organize left-right patterning in C. elegans by uncoupling midline and anteroposterior axis. Dev Cell 2010, 19:402-412.

47. Freytes DO, Wan LQ, Vunjak-Novakovic G: Geometry and force control of cell function. J Cell Biochem 2009, 108:1047-1058.

48. Discher DE, Mooney DJ, Zandstra PW: Growth factors, matrices, and forces combine and control stem cells. Science 2009, 324:1673-1677.

49. Cukierman E, Pankov R, Stevens DR, Yamada KM: Taking cell-matrix adhesions to the third dimension. Science 2001, 294:1708-1712.

doi:10.1186/scrt172

Cite this article as: Wan LQ, et al: Micropatterning of cells reveals chiral morphogenesis. Stem Cell Research \& Therapy 2013, 4:24. 